\title{
Enhancing Classroom Teaching for Students with Speech and Language Exceptionalities: A Social-Emotional Program
}

\author{
Emmanuela Tedone, Krista Muis, Ingrid Sladeczek \\ McGill University, Canada
}

\begin{abstract}
Studies have shown that children with speech and language exceptionalities often have or are at risk of developing poor social and emotional skills [1], [14], [2], [3]. Past research has examined the impact of social communication programs on children with disabilities [4], [5], [6], [7] or focused on cognitive and behavioral skills rather than emotional learning [8]. The current study addressed this gap in the literature by evaluating the effectiveness of a school program in teaching social and emotional learning to children from the ages of four to seven with communication diagnoses. The program was taught over three months and was adapted from Cartledge and Kleefeld's [9] social skills program. Five teachers completed the Taxonomy of Problematic Social Situations for Children [10] or the Preschool Taxonomy of Problem Situations [11], and the Emotion Regulation Checklist [12]. Social and emotional competencies were assessed at two time points. The findings showed that the program was effective at improving Emotion Regulation subscale scores in first grade students. Successful programs contribute to improved performance in social and academic contexts for children with speech and language exceptionalities [2], [3].
\end{abstract}

\section{Introduction}

We are exposed to a myriad of communication cues on a daily basis. These cues encompass verbal and non-verbal form such as, speech, writing, dancing or drawing [13]. All forms of communication allow us to connect events occurring across different timeframes and lay the foundations for success within academic and social environments [13]. Individuals with speech and language exceptionalities often find these environments challenging [14]. There is a comorbidity between speech and language exceptionalities and poor social and emotional skills, mainly because children with these exceptionalities often do not have the communication skills to initiate conversations with peers and teachers, resolve conflicts, ask for help or issue requests [1], [14], [2], [3]. Early detection and intervention programs are shown to be effective at improving social and emotional functioning [1], [15], [13], [14], [16], [2]. Further evidence stems from neuroscience research. The brain areas implicated in these social-emotional processes, namely the prefrontal cortex, temporal-parietal junction, ventromedial prefrontal cortex, dorsolateral prefrontal cortex, orbitofrontal cortex, amygdala, hippocampus and anterior cingulate cortex are easily modified by environmental factors [8]. Given that these brain areas are plastic, interventions directed at building social and emotional skills have the potential to alter neural circuitry [17], [8].

Past researchers focused on children with pragmatic language difficulties [4], [5] or developed specific interventions targeting social communication skills [6], [7]. No studies have been located which target the needs of children aged 4 to 7 with a range of speech and language exceptionalities by combining social and emotional teaching. The current study addressed this gap in the literature by evaluating the effectiveness of a program in teaching socialemotional skills to this aforementioned sample of children. This article will explore the topics relevant to the study. First, emotional and social competencies will be examined to better understand their association and role in typical and atypical speech and language. Next, the need for additional resources and interventions in schools for children with communication exceptionalities will be discussed. These topics will lead into the details of the present study.

\section{Emotional and Social Competencies}

Numerous models of emotional development have been proposed. Saarni's instrumental model is frequently referred to in the literature and incorporates a broad range of emotional skills [1]. Focus is on emotional awareness and understanding, the ability to concisely express how one is feeling and the importance of acknowledging and recognizing that internal emotions are not always consistent with external emotional manifestations [1]. Other models operationalize emotional competencies as consisting of only three related concepts: emotion understanding, emotion regulation and emotion expressiveness [8]. These three concepts are closely linked to one another and serve to enhance an individual's emotional 
experience as a whole [1]. Throughout the process of understanding, regulating and expressing emotions, children use language as a tool to share and reflect on personal experiences [1].

The preschool years are fundamental for the emergence of emotional milestones in typical development [16]. However, children with speech and language exceptionalities tend to struggle with emotional competencies as they are less likely to share a positive or negative emotional experience, understand parental input, resolve misunderstandings or engage in self-talk [1], [2], [17]. If children with these exceptionalities are not taught to self-regulate and emotionally regulate, there is an increase risk of them exhibiting internalizing and externalizing issues in adolescence and adulthood [16].

Social competencies refer to a child's ability to initiate conversation or play, participate in turn-taking activities, understand nonverbal behavior and develop or maintain a relationship with classmates and adults [4], [6]. Children need to be given opportunities to interact with same-age peers, and this generally occurs for the first time during the preschool years. These years lay the foundation for the development of confident children, prepared to engage in academic and social activities and to problem solve [16], [6]. Interacting with peers of the same age naturally pushes children to use their language skills, conflict resolution abilities and perspective taking to initiate and manage social exchanges [16]. For example, a child arguing over Bingo chips with a classmate employs skills in conflict resolution [1]. Social exchanges are more pleasurable and reciprocal when children have effortful control over themselves and their emotional states as connections are formed between peers [17]. Effortful control involves a combination of behaviour regulation and attention regulation, and is also an important factor of emotion regulation [17]. Those with communication exceptionalities are less likely to have effortful control and partake in conversations, and are more likely to become socially withdrawn [1], [2]. These children may believe they are being excluded from their social groups thereby restraining emotions and vulnerabilities, leading to more isolation [17].

\section{Social and Emotional Interventions in Schools}

Coined by the Collaborative for Academic, Social, and Emotional Learning (CASEL), social-emotional learning supports children and adults in the development of critical skills to enhance overall functioning [15]. While critics argue that children should not be given a prescription as to how they need to think and feel, this is not the intended aim of such programs [15]. Rather, emphasis is on the direct teaching of skills through the application of engaging and authentic activities that are "developmentally aligned and sequenced" (p. 23) [15] to model and encourage healthy development.

In a unique study, Stanton-Chapman and colleagues implemented a social communication intervention for eight preschoolers with developmental disabilities [18]. All but one had poor language skills. Initially, the children took part in a play session where they were taught social communication strategies. After practicing these strategies, the children were separated into groups of two, engaged in play and prompted and/or coached by the researchers throughout [18]. In the last session, the children discussed the strategies learned and the vocabulary words used. Language and behaviour during the play sessions were assessed using Stanton-Chapman, Kaiser, Vijay, and CraigUnkefer's (2003) Peer Language and Behavior Code. The researchers found that 6 out of the 8 children displayed an increase in vocabulary as well as peer and verbal requests [18]. These findings offer support to the argument that interventions are effective at facilitating speech and language development [18]. In the preschool and early school years, children are most likely to absorb skills and to thrive within a multitude of contexts [15], [16].

Social and emotional competencies are very closely intertwined [15], [16]. The majority of social situations "involve emotion, and children's ability to be emotionally competent determines how successful they are during their social interactions and relationships" (p. 515) [19]. Children need to understand how to validate and regulate their own emotions before relating to others, while learning to wait their turn, playing cooperatively with peers and problem solving [6], [15], [16]. Those who are unable to deal with emotions in a positive and constructive fashion often become aggressive, withdrawn and display internalizing and 
externalizing problems contributing to rejection and isolation in adolescence and adulthood [13], [2]. School-based interventions serve as a protective factor against these outcomes [15].

\subsection{Aims of the Current Study}

CASEL conducted a meta-analysis of socialemotional programs implemented in schools and isolated several areas of concern: reliability, implementation and validity [15]. More than half of previous studies relied solely on child self-reports to examine impact and program effectiveness [15]. It was also recommended that teachers be observed during regular classroom time to examine whether the tools and skills learned were being applied [15], [17]. Lastly, it appeared that children were not always able to retain and apply the learned skills because of an inability to regulate emotions and think clearly when in social situations [17]. For this reason, teaching emotional learning is a fundamental component of any social skills training [17].

The current study addresses the above concerns. The program was adapted from the program, Taking Part Introducing Social Skills to Children [9]. Guglielmo and Tryon conducted a study to examine the effectiveness of Taking Part at improving social and emotional skills [19]. The researchers focused on two lessons from the fourth unit. Of the students who took part in the study, $86 \%$ expressed that the activities were enjoyable [19]. The teachers also reported that they had observed a positive student response, and results indicated that onethird of students experienced gains in social and emotional skills when the program was combined with classroom reinforcement [19].

The current study was designed to improve students' social and emotional skills within a three-month timespan. These skills were explicitly taught as a group to each grade level through structured and free play, books, modeling, crafts, prompts and reinforcement in the classroom. Contrary to previous interventions, no pull-outs with individual children occurred [18], [7], [17]. The three selected measures, Taxonomy of Problematic Social Situations for Children [10], Preschool Taxonomy of Problem Situations [11], and the Emotion Regulation Checklist [12] were completed by teachers. The first two social measures were chosen because specific social contexts are emphasized instead of a more general assessment of social development [11].
The following was the research question addressed in the study: Is the intervention effective at improving social-emotional competencies in children with speech and language exceptionalities?

\section{Methods}

\subsection{Participants}

Twenty-two children (14 boys, 8 girls) aged 4 to 7 volunteered to participate. Figure 1 illustrates the gender distribution by grade. Of this sample, 5 were enrolled in pre-kindergarten, 12 in kindergarten and the remaining 5 in first grade at an elementary school for students with special needs in Montreal, Canada. In discussion with rehabilitation professionals at the school, the children displaying the greatest need for social-emotional skills were selected to take part in the intervention program. The selected children were chosen as they had a diagnosed speech (e.g. voice, fluency, articulation) and/or language (e.g. phonology, morphology, syntax, intonation, pitch) difficulty. All children received additional in-school therapy from a speech-language pathologist.

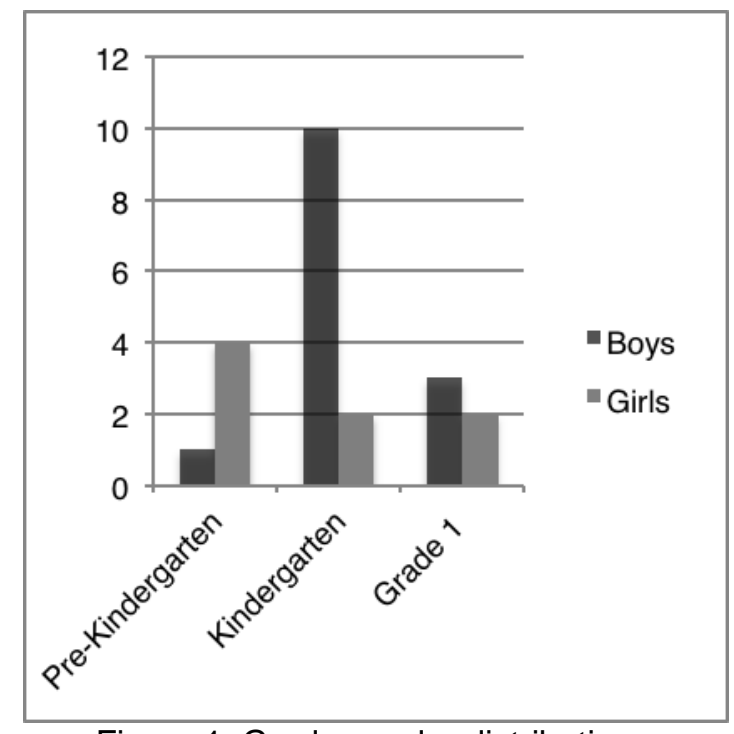

Figure 1. Grade gender distribution

In the previous year, the clinical psychologist at the school conducted a social skills group in specific classrooms. Given that a similar program was integrated in the school curriculum, the current social and emotional program abided by the same regulations. For the current study, all selected students from prekindergarten to first grade participated in the 
intervention program. However, only those who gave assent and whose parents provided authorized consent were included in the research aspect of the project. Students were assigned a random identification number to ensure anonymity and confidentiality.

\subsection{Materials}

4.2.1. Social-emotional program. The socialemotional intervention was largely based on Cartledge and Kleefeld's program, Taking Part Introducing Social Skills to Children [9]. This program was preferred because it is applicable to younger children and those with a variety of special needs [9], [19], [16]. The program was divided into the following six units: (1) Making conversation; (2) Communicating feelings; (3) Expressing oneself; (4) Cooperating with peers; (5) Playing with peers; and, (6) Responding to aggression and conflict. Each unit was then further subdivided into various skills with specific goals, such as smiling or saying hello [9]. The curriculum incorporated questions, skit presentations through the use of puppets and role-plays to practice concepts [9], [19]. Children were encouraged to think about the strategies and use complete sentences when replying to questions with the intention of nurturing executive functions, self-regulation and emotion regulation. Some skills were omitted and adaptations were made to select activities to best meet the childrens' levels. The program was administered by the principal investigator (first author) who has seven years of experience working with students with speech and language exceptionalities and previously assisted in developing interventions as an applied behaviour therapist. An educator employed by the school's rehabilitation centre assisted with the kindergarten group $(n=12)$.

\subsubsection{Taxonomy of Problematic Social} Situations for Children (TOPS). Designed by Dodge and colleagues, the test measure included 44 items organized using a 5-point Likert scale varying from 1 (never) to 5 (almost always) to measure children's social development. The items were created by a group of 50 teachers and 6 clinical psychologists, and organized into six potentially challenging social situations. The focus is on specific scenarios to determine the likelihood of a child's response to particular triggers [10]. The situations are: 1) Peer group entry (when the child has to include himself into a group, 5 items); 2) Response to peer provocation (child maintains integrity when being instigated by other students, 10 items); 3) Response to success (child performs more competently than other students, 3 items); 4) Response to failure (child performs less competently than other students, 9 items); 5) Teacher expectations (concise classroom rules established to teach children social norms, 6 items); and, 6) Social expectations (concise rules established within society to teach children social norms, 11 items) [10].

Each of the six social situations has a high internal consistency ( $\alpha=0.89$ to 0.95 ) and total internal consistency for the measure $(\alpha=0.98)$. Test-retest reliability varied between 0.31 and 0.73 for item scores, 0.57 to 0.72 for each challenging social situation and 0.79 for the final cumulative score [10]. Mean scores can be calculated for each of the six social situations to compare to the total mean for the entire measure, which serves to highlight the social situations that are especially difficult for the children [10]. High scores are indicators for concern [11].

\subsubsection{Preschool Taxonomy of Problem} Situations (PTOPS). Blankemeyer and colleagues developed a preschool version of the Taxonomy of Problematic Social Situations for Children [11]. The measure consists of 60 items and a 5-point Likert scale, ranging from 1 (never) to 5 (almost always) [11]. The items are also categorized by the same six difficult social situation, as well as two additional situations: reactive aggression ( 8 items) and proactive aggression (8 items) [11]. Similar to the TOPS measure, the PTOPS subscales each have a high internal consistency ( $\alpha=0.89$ to 0.95 ) and total internal consistency $(\alpha=0.98)$ [11]. Slight differences exist between the PTOPS and TOPS in that words such as "toy" and "painting a picture" are used instead of "school work" [11]. According to personal communication with Maureen Blankemeyer, the same scoring procedure applies to both measures. No cut-off scores have been determined for clinical or nonclinical samples.

4.2.4. Emotion Regulation Checklist (ERC). Shields and Cicchetti's test measure was used to examine and monitor emotional development [12]. Composed of 24 items, the Emotion Regulation Checklist takes roughly 10 minutes to complete and is rated on a 4-point Likert scale ranging from 1 (almost always) to 4 (never) [12]. It considers positive and negative aspects 
of emotions in situations considered to elicit emotional reactions [6], [20], [12].

The items belong to one of two subscales: Emotion Regulation or Lability/Negativity. The former subscale has 8 items and addresses empathy and emotional understanding (e.g. Is a cheerful child). A score ranging from 8 to 32 reveals superior emotion regulation and emotion expressiveness. Two items are reverse scored. The latter scale has 15 items and captures emotional intensity [20], [12]. In this scale, a score from 15 to 60 implies more emotional dysregulation and negative affect and inflexibility. Four items are reverse scored [20]. The measure has a high internal consistency on the Emotion Regulation ( $\alpha=0.83$ ) subscale and on the Lability/Negativity subscale $(\alpha=0.96)$. There is a significant negative correlation between both subscales $(r=-0.50)$ [12].

\subsection{Procedure}

The primary investigator (first author) began by volunteering in the chosen classrooms for two months prior to obtaining ethics approval to form a bond with the children. Following ethics approval, consent forms were sent to the parents of students selected for the study. Once parental consent was obtained, to ascertain child assent, each child was asked if he or she would like to take part in an activity.

To monitor students' social and emotional development throughout the course of the program, four classroom teachers completed the Taxonomy of Problem Situations for Children (kindergarten and first grade students only) [10] or the Preschool Taxonomy of Problem Situations (pre-kindergarten students only) [11] to measure social competencies and the Emotion Regulation Checklist (all grade levels) [12] to assess emotional competencies. The classroom teachers were chosen because they knew the students well and the scores accurately reflected students' development. A fifth resource teacher completed the measures for eight participants at pretest to establish interrater reliability, which was evaluated using the Pearson correlational coefficient. Significant associations between teacher ratings were revealed. Coefficients ranged from $\mathrm{r}=0.65$ to 0.81 on subscale measures of emotion competency and $r=1$ for both measures of social competencies. The measures were gathered at baseline in the first week of April. Measurements were gathered again upon completion of the program in June. A Wilcoxon paired test was performed to address the research question. The teachers were blind to the purpose of the study.

The intervention occurred in four classrooms for 30 to 45 minutes each on a weekly basis. The primary investigator assisted the teacher and students in the chosen classrooms for an additional six to eight hours per week to apply the social and emotional techniques and skills in more natural settings. In June, the students were compensated for their participation with toys. Teachers were also compensated for their help. A short summary was provided for two parents who asked for information on the activities and findings.

\section{Results}

Preliminary analyses were conducted. The values for skewness and kurtosis for each distribution are illustrated in Table 1.

Table 1. Values of skewness and kurtosis

\begin{tabular}{|l|c|c|}
\hline & Skewness & Kurtosis \\
\hline $\begin{array}{l}\text { Distribution of } \\
\text { differences }\end{array}$ & -0.341 & -1.844 \\
\hline PTOPS & -0.001 & -1.155 \\
\hline TOPS & -0.140 & 1.222 \\
\hline $\begin{array}{l}\text { ERC (Emotion } \\
\text { reg.) }\end{array}$ & 0.168 & 0.413 \\
\hline $\begin{array}{l}\text { ERC } \\
\text { (Lability/Negativity) }\end{array}$ & & \\
\hline
\end{tabular}

The mean and standard deviation for all three measures of social and emotional competencies have been reported in Table 2 and Table 3. The aim was to highlight the childrens' social and emotional levels prior to the intervention to better assess the effectiveness of the program upon completion. Measures of social competency from the Preschool Taxonomy of Problem Situations [11] and the Taxonomy of Problematic Social Situations for Children [10] were in the mid range and considered to be reflective of poorer social development at both pretest and posttest.

Higher scores on the Emotion Regulation subscale of the Emotion Regulation Checklist indicate a greater aptness for emotional expressiveness [20], [12]. Pretest and posttest measurements from this subscale fell into the mid range. 
Table 2. PTOPS/TOPS scores for children at pre and post

\begin{tabular}{|l|c|c|}
\hline & Pretest & Posttest \\
\hline $\begin{array}{l}\text { Grade } \\
\text { levels }\end{array}$ & $\overline{\mathrm{x}}(S D)$ & $\overline{\mathrm{x}}(S D)$ \\
\hline $\begin{array}{l}\text { Pre-K } \\
(n=5) \\
\text { PTOPS }\end{array}$ & $161.8(21.2)$ & $168.4(21.6)$ \\
\hline $\begin{array}{l}\text { Kind. } \\
(n=12) \\
\text { TOPS }\end{array}$ & $63.3(12.8)$ & $77.1(18.9)$ \\
\hline $\begin{array}{l}\text { Gr. 1 } \\
(n=5) \\
\text { TOPS }\end{array}$ & $80.8(20.7)$ & $75.2(22.6)$ \\
\hline
\end{tabular}

However, higher scores on the Lability/Negativity subscale are indicative of inflexibility and mood lability. Pretest and posttest measurements also fell into the mid range. In both cases, mean scores from baseline to post seem to show greater emotional regulation and less lability and negativity, respectively. See Table 3 for the subscale scores according to grade level.

Table 3. ERC subscales scores for children at pre and post

\begin{tabular}{|l|c|c|}
\hline & Pretest & Posttest \\
\hline Grade levels & $\overline{\mathrm{x}}(S D)$ & $\overline{\mathrm{x}}(S D)$ \\
\hline Pre-K $(n=5)$ & & \\
Emotion reg. & $20.6(4.8)$ & $23.6(2.2)$ \\
Lability/Negativity & $32.2(3.9)$ & $29.8(4.8)$ \\
\hline Kinder. $(n=12)$ & & \\
Emotion reg. & $21.7(3.3)$ & $22.3(4.5)$ \\
Lability/Negativity & $24.8(4.0)$ & $24.8(5.3)$ \\
\hline Gr. 1 $(n=5)$ & & \\
Emotion reg. & $22.0(3.6)$ & $24.2(3.6)$ \\
Lability/Negativity & $28.4(5.9)$ & $27.8(4.4)$ \\
\hline
\end{tabular}

A Wilcoxon paired test was conducted, largely due to the small sample size to examine whether each grade level improved from pretest to posttest on measures of social and emotional competencies. An alpha level of 0.05 was used as a threshold for statistical significance.

Results from the Wilcoxon test showed that there was a significant effect of the program on Emotion Regulation subscale scores of the

Emotion Regulation Checklist in Grade 1 students, $p=0.039$. A significant effect of the program on the Taxonomy of Problematic Social Situations for Children scores was also found in kindergarten students, $p=0.010$.
Results also showed that pre-kindergarten students did not significantly improve from pre to post on TOPS scores, $p=0.336$, nor on the ERC for both the Emotion Regulation subscale, $p=0.144$, and the Lability/Negativity subscale, $p=0.066$. The same applies to the kindergarten group for the Emotion Regulation subscale, $p=0.589$ and the Lability/Negativity subscale, $p=0.822$. The first grade Lability/Negativity subscale scores, $p=0.684$ and the TOPS scores, $p=0.175$ also did not differ.

\section{Discussion}

The aim of this study was to assess the effectiveness of a school intervention program in improving the social and emotional skills of young children with speech and language exceptionalities. Elevated scores on all measures at baseline were expected given that the children were selected on the basis of displaying the greatest need for social and emotional learning. At baseline and upon completion of the program, scores on the Preschool Taxonomy of Problem Situations [11] and the Taxonomy of Problematic Social Situations for Children [10] fell into the moderate range and are cause for concern. These scores supported the argument that communication exceptionalities are linked to less-developed social and emotional competencies [6], [14], [2], [3]. Kindergarten students' scores on the Taxonomy of Problematic Social Situations for Children [10] significantly worsened from pretest to posttest which is likely attributable to measurement error, specifically recall bias. One of the kindergarten teachers was away at the end of the school year, and was unable to submit her scores until four months after the end of the program.

All grade levels displayed a large variability in scores on the Preschool Taxonomy of Problem Situations [11] and the Taxonomy of Problematic Social Situations for Children [10] as seen from the large standard deviations across time. These values serve to highlight the importance of differentiated instruction. While each lesson was adapted for each grade level, some children may have benefitted from further adaptions in the teaching of the skills and concepts.

It was surprising to see that the prekindergarten, kindergarten and first grade students displayed an average pretest score that was better than expected on the Emotion Regulation subscale and the Lability/Negativity subscale of the Emotion Regulation Checklist 
[12]. With regards to this checklist, it is important to note that individual scores improved slightly from baseline to the end of the program for all grade levels; however, a significant effect of the program on Emotion Regulation subscale scores was only found in first grade students.

As a whole, these findings can lead to several conclusions. First, the teaching of the program may have been better at fostering emotional development over social development. Second, increasing the teaching frequency to several times per week would give the children more time to practice and generalize the concepts. Last, children with special needs are especially vulnerable and benefit from direct and consistent instruction. Three months is a short period of time to expect to see a pronounced improvement in both social and emotional learning.

There were a couple of limitations. First, no multimethod or multisource assessments were used to examine social and emotional learning at various timepoints, which may affect the reliability and validity of the results. Additional measures, such as Gresham and Elliot's Social Skills Improvement System (SSIS) were initially considered for teachers and parents. The SSIS was deemed to be less applicable to the sample of participants and too time-consuming for teachers to have to complete more than two test measures. Moreover, it was decided that a host of extraneous factors would have to be accounted for if program effectiveness was measured in both home and school contexts. A second limitation was the lack of a control group, which would have provided a point of comparison to assess program effectiveness in improving social-emotional development. Given the restricted timeframe and specific school population, a control group was beyond the scope of this research. Development is monitored at two different times and interrater agreement was incorporated to enhance the reliability and validity of the scientific design.

There were several advantages to the current study. Contrary to prior studies [18], [6], skills and concepts were taught to all the students in each grade level at one time. Also, the measures chosen were indicators of difficulties or successes in specific social and emotional scenarios as opposed to global assessments [11], [10], [12]. Lastly, as pointed out by Suveg and colleagues, the most effective and well-designed programs are developmentally focused [8]. For this reason, the language and activities in
Cartledge and Kleefeld's program [9] was adapted to ensure that the students learned and applied the material.

This project yields theoretical, clinical and practice importance for the area of child development and education since it is the only known study to adapt a program to teach socialemotional competencies to preschool and early school-age children with expressive and receptive language issues. Most children with these issues benefit from the explicit teaching of skills to nurture healthy social and emotional growth through the adaptation and/or development of programs in schools [15], [6], [13], [14], [16], [2]. As an extension to the project, future researchers should run the program for longer to better ascertain effectiveness. It would also be beneficial to conduct follow-up measures six weeks or several months after the program to determine if learning is stable and generalized across multiple contexts.

It is recommended that school psychologists, teachers, educators and speech-language pathologists recognize and address the concerns that often accompany children with speech and language exceptionalities [16], [5], [3]. Through the creation of developmental and age appropriate programs, our knowledge and allocation of resources to the field is advanced.

\section{Acknowledgements}

A huge thank you to my supervisors, the teachers, principal, rehabilitation staff, and my family and friends for their advice, expertise, support, patience and encouragement throughout.

To the children: Their eagerness to learn and positive energy brightened up my day. This project is dedicated to them.

\section{References}

[1] M. Fujiki, B. Brinton, and D. Clarke., "Emotion Regulation in Children with Specific Language Impairment", Language, Speech, and Hearing Services in Schools, American Speech-LanguageHearing Association, United States, 2002, pp. 102111.

[2] L. Rescorla, G. Ross. and S. McClure, "Language Delay and Behavioral/Emotion Problems in Toddles: Findings from Two Developmental Clinics", Journal of Speech, Language, and Hearing Research, American Speech-Language-Hearing Association, United States, 2007, pp. 1063-1078. 
[3] K. Thatcher, K. Fletcher, and B. Decker, "Communications Disorders in the School: Perspectives on Academic and Social Success an Introduction", Psychology in the Schools, Wiley Periodicals Inc, New Jersey, 2008, pp. 579-581.

[4] C. Adams, "Social Communication Intervention for School-Age Children: Rationale and Description", Seminars in Speech and Language, Thieme, New York, 2005, pp. 181-188.

[5] C. Adams, E. Lockton, J. Freed, J. Gaile, G. Earl, K. McBean, M. Nash, J. Green, A. Vail, and J. Law, "The Social Communication Intervention Project: A Randomized Controlled Trial of the Effectiveness of Speech and Language Therapy for School-Age Children who have Pragmatic and Social Communication Problems With or Without Autism Spectrum Disorder", International Journal of Language and Communication, John Wiley and Sons, New Jersey, 2012, pp. 233-244.

[6] T. Stanton-Chapman, and T. Brown, "A Strategy to Increase the Social Interactions of a 3-Year-Old Children with Disabilities in an Inclusive Classroom, Topics in Early Childhood Special Education, SAGE Publications, New York, 2015, pp. 4-14.

[7] T. Stanton-Chapman, C. Denning, and K. Jamison, "Communication Skill Building in Young Children with and without Disabilities in a Preschool Classroom", The Journal of Special Education, SAGE Publications, New York, 2012, pp. 78-93.

[8] C. Suveg, M. Southam-Gerow, K. Goodman, and P. Kendall, "The Role of Emotion Theory and Research in Child Therapy Development", Clinical Psychology: Science and Practice, John Wiley and Sons, New Jersey, 2007, pp. 358-371.

[9] G. Cartledge, and J. Kleefeld, Taking Part: Introducing Social Skills to Children, Research Press Inc, Illinois, 2009.

[10] K.A. Dodge, C.L. McClaskey, and E. Feldman, "Taxonomy of Problematic Social Situations for Children", Duke University,https://childandfamily policy.duke.edu/pdfs/TOPS-Taxonomy-ofProblematic-Social-Situations-for-Children.pdf, 1985.

[11] M. Blankemeyer, R. Culp, L. Hubbs-Tait, and A. Culp, "A Tool for Identifying Preschoolers' Deficits in Social Competence: The Preschool Taxonomy of Problem Situations", Education and Treatment of

Children, West Virginia University Press, West Virginia, 2002, pp. 208-223.

[12] A. Shields, and D. Cicchetti, "Emotion Regulation Checklist", Ohio University, https://etd.ohiolink.edu/.
[13] M. Hunter-Carsch, Y. Tiknaz, P. Cooper, and R. Sage, The Handbook of Social, Emotional and Behavioural Difficulties, Continuum International Publishing Group, United Kingdom, 2006.

[14] G. Malar, N. Sreedevi, and C.B. Suresh, "Trends and Impact of Early Intervention for Communication Disorders at AIISH", Journal of the All India Institute of Speech and Hearing, All India Institute of Speech and Hearing, India, 2013, pp. 173-183.

[15] N. Humphrey, Social and Emotional Learning a Critical Appraisal, SAGE Publications, London, 2013.

[16] P. McCabe, and M. Altamura, "Empirically Valid Strategies to Improve Social and Emotional Competence of Preschool Children", Psychology in the Schools, Wiley Periodicals Inc, New Jersey, 2011, pp. 513-540.

[17] G. Macklem, Practitioner's Guide to Emotion Regulation in School-Aged Children, Springer International Publishing, New York, 2008.

[18] T. Stanton-Chapman, C. Denning, and K. Jamison, "Exploring the Effects of a Social Communication Intervention for Improving Requests and Word Diversity in Preschoolers with Disabilities", Psychology in the Schools, Wiley Periodicals Inc, New Jersey, 2008, pp. 644-664.

[19] H. Guglielmo, and G. Tryon, "Social Skill Training in an Integrated Preschool Program", School Psychology Quarterly, American Psychological Association, Washington, 2001, pp. 158-175.

[20] C. Lincoln, "Mother-child interactions and emotion regulation in preschool children, University of Connecticut, http://digitalcommons.uconn.edu. 